\title{
The Protection Potential of an Alum-Adjuvanted F1 Protein in Dry Powder against Plague in Mice
}

\author{
Wang Wang, Yiran Liu, Xiangna Zhao, Zongmin Du, Yafang Tan, Yujing Bi, Yanping Han, Ruifu Yang* and Xiaoyi Wang* \\ Laboratory of Analytical Microbiology, State Key Laboratory of Pathogen and Biosecurity, Beijing Institute of Microbiology and Epidemiology, Beijing 100071, China
}

\begin{abstract}
Aluminum hydroxide is the only adjuvant for use in human vaccines. The commercial alum-adjuvanted vaccines are formulated as liquid without exception. We prepared an alum-adjuvanted dry powder vaccine and demonstrated that it provided good protection for plague. The preparation strategy is simple and effective, and can be carried out in a large scale. The dry powder vaccine can be quickly reconstituted using sterile water for injection. The dry powder vaccines have potential advantages over liquid vaccines, including good stability and extended shelf life.
\end{abstract}

Keywords: F1 antigen; Plague; Protection; Dry powder vaccine; $Y$. pestis

\section{Introduction}

Plague is a zoonotic disease caused by Gram-negative bacterium Yersinia pestis, which is usually transmitted to humans from infected rodents via the bite of an infected flea [1]. Historically, plague has been an awful infectious disease afflicting human populations, leading to millions of deaths. Plague has been recently classified as a re-emerging disease by the World Health Organization [2] and has attracted a considerable attention because of its potential use as an agent of biological warfare or bioterrorism [3].

To date, there is not an ideal plague vaccine for human use. Killed whole cell vaccines offer only short-term protection against bubonic plague and frequent boosting is needed to maintain immunity [4]. Live attenuated vaccine EV76 was effective against bubonic and pneumonic plague, but it showed side effects of varying severity and was not used in the western world [4]. Multiple DNA vaccines based on $Y$. pestis $\mathrm{F} 1$ and $\mathrm{LcrV}$ antigens alone or in combination were efficacious against both bubonic and pneumonic plague [5-11]. However, DNA vaccines usually elicit lower and slower immune responses than conventional vaccines, and gene gun immunization that delivers DNA-coated particles into the dermis of the skin needs to be used for optimal immune responses [10-12]. In contrast, subunit vaccines have potential advantages over the traditional vaccines (killed whole cell vaccine and live attenuated vaccine) and DNA vaccines in terms of safety or efficacy against $Y$. pestis. It has been demonstrated that $\mathrm{F} 1$ and LcrV antigens used alone or in combination can protect mice against bubonic and pneumonic plague [13].

Currently, an alum-adjuvanted vaccine formulation comprising the F1 and LcrV antigens and an engineered F1-LcrV fusion protein was developed by the United Kingdom's Ministry of Defense and the United States Army Medical Research Institute of Infectious Diseases, respectively. Both vaccine candidates began phase one clinical trials in 2005 [14]. In our previous study, the native F1 antigen was extracted from $Y$. pestis EV76 [15] and an LcrV variant containing amino acids 1 to 270 of LcrV (rV270) was prepared from engineered Escherichia coli BL21 [16]. Our study demonstrated that the alum-adjuvanted subunit vaccine comprising the native $\mathrm{F} 1$ and rV270 provided effective protection in mice, guinea pigs and rabbits [17]. Generally, the alum-adjuvanted vaccines are in the form of colloidal state, and can not be lyophilized or frozen for stabilization because freezing or freeze drying them often causes immunogenicity loss [18]. Recently, an alum-adjuvanted dry powder vaccine containing a recombinant
F1-V fusion protein of $Y$. pestis provided an equivalent efficacy to the corresponding liquid formulation for protection against $Y$. pestis challenge in a mouse model [19]. In the present study, an alumadjuvanted dry powder vaccine comprising F1 antigen and mannitol was prepared by a simple vacuum-drying strategy, and evaluated for protective efficacy against $Y$. pestis challenge in mouse model after it was stored at $40^{\circ} \mathrm{C}$ for 10 days.

\section{Materials and Methods}

\section{Animal}

Female BALB/c mice of 6-8 weeks were used in this study. All the animals were group-housed and provided with food and fresh water ad libitum during the entire course of this study. All animal experiments were conducted strictly in compliance with the Regulations of Good Laboratory Practice for nonclinical laboratory studies of drug issued by the National Scientific and Technologic Committee of People's Republic of China.

\section{Vaccines}

The native $\mathrm{F} 1$ antigen was prepared from $Y$. pestis EV76 by physical disruption, followed by a combination of ammonium sulfate fractionation and Sephacryl S-200HR column filtration chromatography [15]. The native F1 antigen was adsorbed to $25 \%$ $(\mathrm{v} / \mathrm{v})$ aluminum hydroxide gel (the Lanzhou Institute of Biological Products, China) in PBS buffer to give the alum-containing liquid vaccine formulations (designated LV20, LV10 and LV5 in this report, respectively) containing 20,10 and $5 \mu \mathrm{g}$ of $\mathrm{F} 1$ antigen in a final volume

*Corresponding authors: Ruifu Yang, Professor, Laboratory of Analytical Microbiology, State Key Laboratory of Pathogen and Biosecurity, Beijing Institute of Microbiology and Epidemiology, Beijing 100071, China, Fax: 86-10-63815689; E-mail: ruifuyang@gmail.com

Xiaoyi Wang, Associate Professor, Laboratory of Analytical Microbiology, State Key Laboratory of Pathogen and Biosecurity, Beijing Institute of Microbiology and Epidemiology, Beijing 100071, China, Fax: 86-10-63815689; E-mail: wgenome@ yahoo.com.cn

Received October 21, 2010; Accepted November 11, 2010; Published Novembe 13,2010

Citation: Wang W, Liu Y, Zhao X, Du Z, Tan Y, et al. (2010) The Protection Potentia of an Alum-Adjuvanted F1 Protein in Dry Powder against Plague in Mice . J Vaccines Vaccin 1: 105. doi:10.4172/2157-7560.1000105

Copyright: @ 2010 Wang W, et al. This is an open-access article distributed unde the terms of the Creative Commons Attribution License, which permits unrestricted use, distribution, and reproduction in any medium, provided the original author and source are credited. 
of $100 \mu$ l, respectively. The corresponding dry powder vaccine was prepared by dissolving $5 \mathrm{mg}$ of mannitol in $100 \mu \mathrm{l}$ of alum-containing liquid vaccine and then vacuum drying at room temperature for $24 \mathrm{~h}$ with the help of drying agent calcium chloride. These dry-powder vaccines (designated DV20, DV10 and DV5 in this study, respectively) were placed in $40^{\circ} \mathrm{C}$ incubator for 10 days before they were reconstituted with sterile distilled water for immunizing mice.

\section{Animal immunizations}

Seventy BALB/c mice were randomly divided into 7 groups at 10 mice per group. Mice in groups 1-3 were immunized intramuscularly with $100 \mu \mathrm{l}$ of sterile distilled water-reconstituted DV20, DV10 and DV5, respectively. Animals in groups 4-6 were given the corresponding LV20, LV10 and LV5, respectively. Those in group 7 were injected the same volume of aluminum hydroxide in PBS buffer $(25 \%, \mathrm{v} / \mathrm{v})$, as controls. After primary immunization, on day 21 the animals were boosted with an identical dose at the same injection sites.

\section{ELISA for detection of F1-specific IgG titer and IgG subclasses}

Sera collected from immunized animals on week 8 after primary immunizations were assayed for the presence of anti-F1-specific IgG by a modified ELISA. Briefly, 96-well microtitre plates were coated with $\mathrm{F} 1$ antigen diluted to $500 \mathrm{ng} / \mathrm{ml}$ in $0.06 \mathrm{M}$ sodium carbonate buffer ( $\mathrm{pH} 9.6)$ and incubated for overnight at $4^{\circ} \mathrm{C}$. Non-specific binding was blocked with $0.1 \%$ casein in $0.01 \mathrm{M}$ phosphate-buffered saline. Test sera were added to plates with serial dilution in $0.01 \mathrm{M}$ PBS buffer containing $0.05 \%$ casein and incubated for $30 \mathrm{~min}$ at $37^{\circ} \mathrm{C}$. After five washes with $0.01 \mathrm{M}$ PBS buffer containing $0.05 \%$ Tween 20 $(\mathrm{pH} 7.2), 100 \mu \mathrm{l}$ of secondary antibodies conjugated to horseradish peroxidase was added to each well and incubated for $20 \mathrm{~min}$ at $37^{\circ} \mathrm{C}$. The plates were washed three times in $0.01 \mathrm{M}$ PBS buffer containing $0.05 \%$ Tween $20(\mathrm{pH} 7.2)$ and $100 \mathrm{l}$ of $0.01 \%$ peroxidase substrate 3 , 3', 5, 5'-tetramethylbenzidine (TMB) was added to each well. The reaction was stopped by the addition of $50 \mu \mathrm{l}$ of $2.5 \mathrm{M} \mathrm{H}_{2} \mathrm{SO}_{4}$ per well, and then optical density (OD) was read at $450 \mathrm{~nm}$ with an ELISA plate reader (BIO-RAD). An IgG1 or IgG2a or IgG2b isotype-specific ELISA was conducted as described above, using horseradish peroxidaseconjugated goat anti-mouse $\operatorname{IgG} 1$ or $\operatorname{IgG} 2 \mathrm{a}$ or $\operatorname{IgG} 2 \mathrm{~b}$ (Sigma-Aldrich). The titre of antibody was estimated as the maximum dilution of serum giving an OD reading above 0.2 units over background. Background values were obtained from serum samples collected from the animals only receiving aluminum hydroxide. Antibody endpoint titre per immunization group is presented as the geometric mean endpoint titre to F1.

\section{Challenge with $Y$. pestis}

The immunized animals were challenged $s$. $c$. with $1320 \mathrm{CFU} Y$. pestis strain 201 that has a median lethal dose (MLD) of 3.3 colonyforming units (CFU) for BALB/C mice by the subcutaneous route, and then closely observed for 14 days. All the survival animals were killed humanely for a post-mortem examination. Cardiac blood, livers, spleens, lungs and lymph nodes of the challenged animals were removed to confirm if $Y$. pestis was present in these organs.

\section{Statistical analysis}

The geometric mean of antibody titres and the standard error of the means were calculated from the data obtained by ELISA. The difference of the data among groups was compared by analysis of variance (ANOVA) with SARS 8.0 software. A Log-Rank Test analysis was used to determine the difference of protective efficacy between treatment groups. Probability values of $<0.05$ were taken as significant.

\section{Results}

\section{Antibody responses to $\mathrm{F} 1$ antigen}

Sera from the immunized mice with both dry powder and liquid vaccines all developed very high titers of anti-F1-specific IgG, and no anti-F1 IgG was detected in all the control animals (Figure 1). ANOVA analysis demonstrated that there was no antibody titer difference among six groups of mice immunized with DV20, DV10, DV5, LV20, LV10 and LV5 $(F=0.21, p>0.05)$, suggesting that the immune response may have been saturated at the dose level of $5 \mu \mathrm{g}$ F1 in mice. Analysis of $\operatorname{Ig} G$ subclasses of mice receiving the vaccines DV20, DV10, DV5, LV20, LV10 and LV5 showed a predominant response for $\operatorname{IgG} 1$ and a lesser response for $\operatorname{lgG} 2 \mathrm{a}$ and $\operatorname{lgG} 2 \mathrm{~b}$, suggesting a predominant $\mathrm{Th} 2$ response (data not shown).

\section{Observation of protective efficacy}

Effective protection was observed for all the animals immunized with different concentrations of both forms of vaccine by challenging with $4 \times 10^{2}$ LD50 $Y$. pestis strain 201 by the subcutaneous route, whereas all the control mice succumbed to the same dose of $Y$. pestis 201 challenge (Table 1). Survival rates in mice vaccinated with the dry powder vaccines were similar to those resulting from administration of standard liquid vaccines. The reconstituted powders administered by i.m. routes provided animals with survival rates of 50 to $60 \%$, while the original liquid formulations protected animals at survival rates of 50 to $70 \%$. A Log-Rank Test analysis showed no significant survival difference among six groups of mice immunized with DV20, DV10, DV5, LV20, LV10 and LV5 ( $p>0.05)$. This result indicated that the

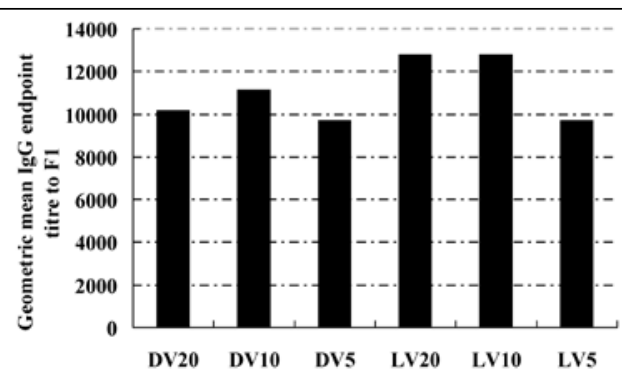

Figure 1: The geometric mean values of $\mathrm{lgG}$ titer to $\mathrm{F} 1$ were derived from ten serum samples per group. DV stands for dry vaccine and LV for liquid vaccine; the numbers following DV or LV represent concentration of 20,10 and $5 \mu \mathrm{g}$ of $\mathrm{F} 1$ antigen in a final volume of $100 \mu \mathrm{l}$, respectively. Standard deviation (SD) corresponding to the groups of DV20, DV10, DV5, LV20, LV10 and LV5 was 1.6, 2.1, 2.5, 1.6, 2.3 and 2.5, respectively.

\begin{tabular}{|l|l|l|}
\hline Treatment groups & Challenge dose (LD50) & Survival/total \\
\hline${ }^{*}$ DV20 & $4 \times 10^{2}$ & $5 / 10$ \\
\hline DV10 & $4 \times 10^{2}$ & $6 / 10$ \\
\hline DV5 & $4 \times 10^{2}$ & $5 / 10$ \\
\hline${ }^{* *}$ LV20 & $4 \times 10^{2}$ & $7 / 10$ \\
\hline LV10 & $4 \times 10^{2}$ & $6 / 10$ \\
\hline LV5 & $4 \times 10^{2}$ & $5 / 10$ \\
\hline Alum alone & $4 \times 10^{2}$ & $0 / 10$ \\
\hline
\end{tabular}

*: DV stands for dry vaccine and the numbers following DV represent concentration of 20,10 and $5 \mu \mathrm{g}$ of $\mathrm{F} 1$ antigen in a final volume of $100 \mu \mathrm{l}$, respectively.

**: LV stands for liquid vaccine, and the numbers following LV represent concentration of 20,10 and $5 \mu \mathrm{g}$ of $\mathrm{F} 1$ antigen in a final volume of $100 \mu \mathrm{l}$, respectively.

Table 1: Protection of mice against challenge with Y. pestis. 
dry powder vaccines provided similar protection to the liquid ones, and that there was no dose-dependent protective efficacy within the

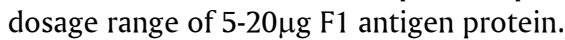

\section{Analysis of tissue pathogen load}

A post-mortem was carried out on all animals who succumbed to the challenge in the 14 days post-challenge observation period, and $Y$. pestis were recovered from the cardiac blood, livers, spleens, lungs and lymph nodes of all dead animals. This result indicated that the death of animals was caused by the systemic infection of $Y$. pestis. At day 14 post-challenge, survivors were killed humanely and autopsied for post-mortem analysis. Microbiological analysis did not isolate $Y$. pestis from cardiac blood, livers, spleens, lungs and lymph nodes of immunized animals, indicating that the bacteria have been eradicated from mice.

\section{Discussion}

In this study, we developed a simple and efficient strategy for preparing an alum-adjuvanted native F1 dry powder vaccine and demonstrated that the dry powder vaccine elicited serum antibody responses and provided protection for plague at levels equivalent to that of a standard liquid formulation of $\mathrm{F} 1$ antigen. Analysis of $\operatorname{IgG}$ subclasses of mice receiving the vaccines DV20, DV10, DV5, LV20, LV10 and LV5 showed a predominant response for IgG1 and a lesser response for $\lg G 2 a$ and $\lg G 2 b$, suggesting that both dry powder vaccines and liquid vaccines induced a predominant Th2 humoral response. The commercial alum-adjuvanted vaccines are formulated as liquid without exception, because the freezing or freeze drying on them generally causes immunogenicity loss. However, in the aqueous environment they are subjected to physical and chemical effects that may lead to inactivation of antigen proteins. Elevated temperature increases the rate of inactivation of antigen proteins, whereas temperature below the freezing point often causes immunogenicity loss of alum-adjuvanted vaccines [20]. Therefore, the commercial alum-adjuvanted liquid vaccines have to be stored within the narrow temperature range of 2 to $8^{\circ} \mathrm{C}$, which makes the process of distribution and storage complicated and expensive.

Recently, a spray-freeze-dried powder vaccine containing a recombinant $\mathrm{F} 1-\mathrm{V}$ fusion protein of $Y$. pestis was evaluated for protection against $Y$. pestis challenge in a mouse model. The results showed that the immunized mice with powder vaccine by intramuscular injection developed specific $\lg G$ responses and provided $70-90 \%$ protection against $1 \times 10^{3}$ LD50 of $Y$. pestis challenged subcutaneously, at a level equivalent to those immunized with the corresponding liquid formulation [19]. Recently, dry powder vaccines have attracted much attention due to their potential advantages over conventional liquid vaccines, including improved stability and extended shelf life [21-23]. In the present study, preparation of an alum-adjuvanted native $\mathrm{F} 1$ antigen dry powder vaccine was achieved by simply dissolving mannitol in alumadjuvanted native $\mathrm{F} 1$ antigen liquid formulation and vacuum drying with the help of calcium chloride at room temperature. As revealed in this study, the preparation strategy is simple and effective, and can be carried out on a large scale. The dry powder vaccine can be quickly reconstituted using sterile distilled water for injection. Mouse tests revealed that the dry powder vaccine elicited similar IgG titer to F1 with corresponding liquid vaccine and provided the same protection as the liquid formulation against subcutaneous challenge with $4 \times 10^{2}$ LD50 of $Y$. pestis strain 201. Compared with a spray-freeze-dried powder vaccine preparation, this preparation strategy is simple, cheap and does not need expensive equipments, and is suit for use in developing countries.
Generally, conversion of biologically active protein solution into a dry powder formulation using suitable excipients and procedures results in more stable products that can be stored at room temperature for longer time. In addition, a dry powder vaccine can be stored below the freezing point without causing the formation of ice which may damage antigens [24]. To investigate the stability of the dry powder vaccine, we once tried to determine the concentration of F1 antigen in the dry powder vaccine by ELISA. However, our results indicated that $\mathrm{F} 1$ antigen was difficult to be released from F1-adsorbed alum adjuvant (data not shown). Hence, we have to use indirectly antibody response and protective efficacy to evaluate the stability of the dry powder vaccines. The dry powder vaccines were first incubated at $40^{\circ} \mathrm{C}$ for 10 days, and then used to immunize mice together with new prepared liquid vaccines. The current results indicate that reconstituted dried vaccines of native F1 antigen elicit similar serum antibody titre to the corresponding liquid ones and provide protection against $Y$. pestis at levels equivalent to those of the corresponding liquid formulation. These results demonstrated that conversion of native F1 alum-adjuvanted liquid vaccine into a dry powder formulation using mannitol and vacuum-drying process did not decrease the antibody response and protection efficacy against $Y$. pestis challenge even though the dry vaccines were stored at $40^{\circ} \mathrm{C}$ for 10 days. These results suggest that alum-adjuvanted dry powder vaccines have similar efficacy to the corresponding liquid ones, and might provide an alternative to traditional liquid formulation.

\section{Acknowledgement}

This work was supported by the National Natural Science Foundation of China (contract no. 30771920) and the National Key Program for Infectious Diseases of China (2008ZX10004-015).

\section{Reference}

1. Perry RD, Fetherston JD (1997) Yersinia pestis--etiologic agent of plague. Clin Microbiol Rev 10: 35-66.

2. Williamson ED (2001) Plague vaccine research and development. J Appl Microbiol 91: 606-608.

3. Riedel S (2005) Plague: from natural disease to bioterrorism. Proc (Bayl Univ Med Cent) 18: 116-124

4. Russell P, Eley SM, Hibbs SE, Manchee RJ, Stagg AJ, et al. (1995) A comparison of Plague vaccine, USP and EV76 vaccine induced protection against Yersinia pestis in a murine model. Vaccine 13: 1551-1556.

5. Yang X, Hinnebusch BJ, Trunkle T, Bosio CM, Suo Z, et al. (2007) Ora vaccination with salmonella simultaneously expressing Yersinia pestis F1 and $\mathrm{V}$ antigens protects against bubonic and pneumonic plague. J Immunol 178: 1059-1067.

6. Palin A, Chattopadhyay A, Park S, Delmas G, Suresh R, et al. (2007) An optimized vaccine vector based on recombinant vesicular stomatitis virus gives high-level, long-term protection against Yersinia pestis challenge. Vaccine 25: 741-750.

7. Liu WT, Hsu HL, Liang CC, Chuang CC, Lin HC et al. (2007) A comparison of immunogenicity and protective immunity against experimental plague by intranasal and/or combined with oral immunization of mice with attenuated Salmonella serovar Typhimurium expressing secreted Yersinia pestis F1 and V antigen. FEMS Immunol Med Microbiol 51: 58-69.

8. Garmory HS, Griffin KF, Brown KA, Titball RW (2003) Oral immunisation with live aroA attenuated Salmonella enterica serovar Typhimurium expressing the Yersinia pestis $\mathrm{V}$ antigen protects mice against plague. Vaccine 21: 3051-3057.

9. Wang S, Heilman D, Liu F, Giehl T, Joshi S, et al. (2004) A DNA vaccine producing LcrV antigen in oligomers is effective in protecting mice from lethal mucosal challenge of plague. Vaccine 22: 3348-3357.

10. Garmory HS, Freeman D, Brown KA, Titball RW (2004) Protection agains plague afforded by immunisation with DNA vaccines optimised for expression of the Yersinia pestis $V$ antigen. Vaccine 22: 947-957. 
Citation: Wang W, Liu Y, Zhao X, Du Z, Tan Y, et al. (2010) The Protection Potential of an Alum-Adjuvanted F1 Protein in Dry Powder against Plague in Mice. J Vaccines Vaccin 1: 105. doi:10.4172/2157-7560.1000105

11. Grosfeld H, Cohen S, Bino T, Flashner Y, Ber R, et al. (2003) Effective Protective Immunity to Yersinia pestis Infection Conferred by DNA Vaccine Coding for Derivatives of the F1 Capsular Antigen. Infect Immun 71: 374-383.

12. Bennett AM, Phillpotts RJ, Perkins SD, Jacobs SC, Williamson ED (1999) Gene gun mediated vaccination is superior to manual delivery for immunisation with DNA vaccines expressing protective antigens from Yersinia pestis or Venezuelan Equine Encephalitis virus. Vaccine 18: 588-596.

13. Leary SE, Griffin KF, Garmory HS, Williamson ED, Titball RW (1997) Expression of an F1/V fusion protein in attenuated Salmonella typhimurium and protection of mice against plague. Microb Pathog 23: 167-179.

14. Anisimov AP, Lindler LE, Pier GB (2004) Intraspecific diversity of Yersinia pestis. Clin Microbiol Rev 17: 434-464.

15. Wang T, Qi Z, Wu B, Zhu Z, Yang Y, et al. (2008) A new purification strategy for fraction 1 capsular antigen and its efficacy against Yersinia pestis virulent strain challenge. Protein Expr Purif 61: 7-12.

16. Wang T QZ, Wu BC, Zhu ZW, Yang YH, Cui BZ, et al. (2008) Cloning and Expression of Yersinia pestis rV270 Antigen and Efficacy against Y. pestis Virulent Strain Challenge. Letters in Biotechnology 19: 663-666.

17. Qi Z, Zhou L, Zhang Q, Ren L, Dai R, et al. (2010) Comparison of mouse, guinea pig and rabbit models for evaluation of plague subunit vaccine F1 + rV270. Vaccine 28: 1655-1660.
18. Maa YF, Zhao L, Payne LG, Chen D (2003) Stabilization of alum-adjuvanted vaccine dry powder formulations: mechanism and application. J Pharm Sci 92: 319-332.

19. Huang J, D'Souza AJ, Alarcon JB, Mikszta JA, Ford BM, et al. (2009) Protective Immunity in Mice Achieved with Dry Powder Formulation and Alternative Delivery of Plague F1-V Vaccine. Clin Vaccine Immunol 16: 719-725.

20. Diminsky D, Moav N, Gorecki M, Barenholz Y (1999) Physical, chemical, and immunological stability of CHO-derived hepatitis B surface antigen (HBsAg) particles. Vaccine 18: 3-17.

21. Huang J, Garmise RJ, Crowder TM, Mar K, Hwang CR, et al. (2004) A nove dry powder influenza vaccine and intranasal delivery technology: induction of systemic and mucosal immune responses in rats. Vaccine 23: 794-801.

22. Jiang G, Joshi SB, Peek LJ, Brandau DT, Huang J, et al. (2006) Anthrax vaccine powder formulations for nasal mucosal delivery. J Pharm Sci 95: 80-96.

23. Sullivan VJ, Mikszta JA, Laurent P, Huang J, Ford B (2006) Noninvasive delivery technologies: respiratory delivery of vaccines. Expert Opin Drug Deliv 3: 87-95.

24. Amorij JP, Meulenaar J, Hinrichs WL, Stegmann T, Huckriede A, et al. (2007) Rational design of an influenza subunit vaccine powder with sugar glass technology: Preventing conformational changes of haemagglutinin during freezing and freeze-drying. Vaccine 25: 6447-6457. 